\title{
TORELLI THEOREM FOR THE MODULI SPACE OF PARABOLIC HIGGS BUNDLES
}

\author{
TOMAS L. GÓMEZ AND MARINA LOGARES
}

\begin{abstract}
In this article we extend the proof given by Biswas and Gómez $B \mathrm{~B}$. of a Torelli theorem for the moduli space of Higgs bundles with fixed determinant, to the parabolic situation.
\end{abstract}

\section{INTRODUCTION}

The classical Torelli theorem says that if two smooth compact Riemann surfaces have Jacobians which are isomorphic as polarized varieties, then the Riemann surfaces are isomorphic. In other words, a smooth compact Riemann surface can be recovered from its principally polarized Jacobian. Analogously, in [MN] Mumford and Newstead proved that a smooth complex projective curve $X$ can be recovered from the isomorphism class of $M_{X}^{2, \xi}$, the moduli space of stable vector bundles over $X$ with rank 2 and fixed determinant $\xi$ of odd degree. This result was generalized by Narasimhan and Ramanan in [NR] where they extended it for any rank. They

show that the intermediate Jacobian of $M_{X}^{r, \xi}$ has a polarization given by the positive generator of $\operatorname{Pic}\left(M_{X}^{r, \xi}\right)$. This polarized intermediate Jacobian is isomorphic to the Jacobian of the curve $X$, so they reduce their proof to the classical Torelli theorem.

Parabolic structures on holomorphic vector bundles were introduced by Mehta and Seshadri in [MS] inspired by the work of Weil. In [W] Weil studied the problem of characterizing fiber bundles which arise from a representation of the fundamental group. The parabolic situation corresponds to a representation of the fundamental group of the complement of a finite set of marked points on a Riemann surface with prescribed holonomy around the marked points. Mehta and Seshadri constructed its moduli space using Geometric Invariant Theory in [MS].

Higgs bundles appear when we consider non unitary representations of the fundamental group, for instance representations into $\mathrm{GL}(n, \mathbb{C})$. Hitchin started the study of those in [H1] and gave the moduli space the structure of a completely integrable Hamiltonian system in [H2].

In $[\mathrm{BBB}$, Balaji, del Baño and Biswas prove a Torelli theorem for parabolic bundles of rank 2, and in [BG], Biswas and Gómez give a Torelli theorem for Higgs bundles. Our goal will be to provide the moduli space of parabolic Higgs bundles with a Torelli type theorem.

In this article we prove the following (see section 2 for notation):

2000 Mathematics Subject Classification. Primary 14D22, Secondary 14D20.

Key words and phrases. Parabolic Higgs bundles, Hitchin map, spectral curve.

T.G. supported by the Spanish Ministerio de Educación y Ciencia [MTM2007-63582] M.L. supported by a postdoctoral grant at MPIM-Bonn and the grant SFRH/BPD/27039/2006 by F.C.T. and C.M.U.P. (Portugal) through the programmes POCTI and POSI, with national and European Community structural funds. 
Theorem 1.1. Let $X$ and $X^{\prime}$ be Riemann surfaces of genus $g \geq 2$, let $\mathcal{N}_{\xi}(2, d, \alpha)$ and $\mathcal{N}_{\xi}^{\prime}(2, d, \alpha)$ be the moduli spaces of parabolic Higgs bundles over $X$ and $X^{\prime}$ respectively, with fixed determinant $\xi$ of odd degree $d$. If there is an isomorphism between $\mathcal{N}_{\xi}(2, d, \alpha)$ and $\mathcal{N}_{\xi}^{\prime}(2, d, \alpha)$ then there is an isomorphism between $X$ and $X^{\prime}$ sending the marked points at $X$ to the marked points at $X^{\prime}$.

Note that we have to assume that the rank is 2, because we apply the Torelli theorem of $[\mathrm{BBB}$. Before proving the theorem, we need to do a detailed analysis of the moduli space of parabolic Higgs bundles, and this is done for any rank $r>1$ and degree $d$ coprime to $r$.

In [BG, it is shown that the moduli space of vector bundles, which is embedded naturally inside the moduli space of Higgs bundles as Higgs bundles with zero Higgs field, can be characterized intrinsically. In other words, given the moduli space of Higgs bundles as an abstract variety, we can find the subvariety which corresponds to Higgs bundles with zero Higgs field, and then we can apply the Torelli theorem for the moduli space of vector bundles.

Following the ideas of [BG], given the moduli space of parabolic Higgs bundles as an abstract algebraic variety, we first recover the Hitchin map. In section 5 we use the Kodaira-Spencer map and study the deformations of some objects related to the Hitchin map to show that any $\mathbb{C}^{*}$-action admitting a lift to the moduli space of parabolic Higgs bundles, has the origin as fixed point. Since the standard $\mathbb{C}^{*}$-action on the Hitchin space induced by sending a Higgs pair $(E, \Phi)$ to $(E, t \Phi)$ has the origin as the unique fixed point, we recover the origin of the Hitchin space.

By definition, the fiber over this point is the nilpotent cone, and then we show that the only irreducible component of the nilpotent cone which does not admit a nontrivial $\mathbb{C}^{*}$-action is the component corresponding to parabolic bundles. Therefore, we have identified the moduli space of parabolic bundles as a subvariety, and then we can apply $[\mathrm{BBB}]$ to recover the curve and the marked points.

\section{Preliminaries}

Let $X$ be a smooth projective curve over $\mathbb{C}$ of genus $g \geq 2$. Let $D$ be a finite set of $n \geq 1$ distinct points of $X$. A parabolic vector bundle over $X$ is a holomorphic vector bundle of rank $r$ (we assume $r \geq 2$ ) together with a weighted flag on the fiber over each $x \in D$, called parabolic structure, that is

$$
\begin{gathered}
E=E_{x, 0} \supset E_{x, 1} \supset \cdots \supset E_{x, r} \supset\{0\} \\
0 \leq \alpha_{1}(x)<\cdots<\alpha_{r}(x)<1 .
\end{gathered}
$$

The parabolic structure is said to have full flags whenever each step of the filtration has dimension one, i.e. $\operatorname{dim}\left(E_{x, i} / E_{x, i+1}\right)=1$. We denote $\alpha=\left\{\left(\alpha_{1}(x), \ldots, \alpha_{r}(x)\right)\right\}_{x \in D}$ to the system of weights corresponding to a fixed parabolic structure.

The parabolic degree of a parabolic vector bundle is defined as

$$
\operatorname{pardeg}(E)=\operatorname{deg}(E)+\sum_{i, x \in D} \alpha_{i}(x),
$$

and the parabolic slope is then $\operatorname{par} \mu(E)=\operatorname{pardeg}(E) / r k(E)$.

A parabolic bundle is said to be (semi)-stable if for all parabolic subbundles, that is holomorphic subbundles $F \subset E$ with the induced parabolic structure, the following condition for the parabolic slope is satisfied

$$
\operatorname{par} \mu(F)<\operatorname{par} \mu(E) \quad(\leq) .
$$


The moduli space $\mathcal{M}(r, d, \alpha)$ of semistable parabolic vector bundles of rank $r$ and degree $d$ was constructed by Mehta and Seshadri using Mumford's geometric invariant theory in $[\mathrm{MS}$. They also proved that $\mathcal{M}(r, d, \alpha)$ is a normal projective variety of dimension

$$
r^{2}(g-1)+1+\frac{n\left(r^{2}-r\right)}{2},
$$

where the last summand comes from the fact that the flags we are considering over each point of $D$ are full flags. Moreover, it is smooth for a generic choice of weights, where the system of weights $\alpha$ is generic whenever for such weights semistability implies stability. From now on we assume the parabolic structure to have full flags and generic weights.

Let $\xi$ be a line bundle on $X$. We denote $\mathcal{M}_{\xi}$ the moduli space of stable parabolic vector bundles with fixed determinant. It is a projective scheme of dimension

$$
(g-1)\left(r^{2}-1\right)+\frac{n\left(r^{2}-r\right)}{2} .
$$

A parabolic Higgs bundle $(E, \Phi)$ is a parabolic vector bundle $E$ together with a homomorphism, called Higgs field,

$$
\Phi: E \longrightarrow E \otimes K(D)
$$

such that it is a strongly parabolic homomorphism, i.e. for each point $x \in D$, the homomorphism induced in the fibers satisfies

$$
\left.\Phi\left(E_{x, i}\right) \subset E_{x, i+1} \otimes K(D)\right|_{x}
$$

where $K$ is the canonical bundle over $X$, and $K(D)$ denotes the line bundle $K \otimes$ $\mathcal{O}_{X}(D)$.

A parabolic Higgs bundle is called (semi)-stable whenever the slope condition holds for all $\Phi$-invariant parabolic subbundles, that is $F \subset E$ parabolic subbundle, such that $\Phi(F) \subset F \otimes K(D)$.

Denote $\mathcal{N}(r, d, \alpha)$ the moduli space of stable rank $r$ degree $d$ and weight type $\alpha$ parabolic Higgs bundles. It contains as an open subset the cotangent bundle of the moduli space of stable parabolic vector bundles and, under the assumption of genericity for the weights, it is a smooth irreducible complex variety of dimension

$$
\operatorname{dim} \mathcal{N}(r, d, \alpha)=r^{2}(2 g-2)+2+n\left(r^{2}-r\right)
$$

where $n$ is the number of marked points on $X$. Indeed, let $[E] \in \mathcal{M}(r, d, \alpha)$. Denote by SParEnd $(E)$ the sheaf of strongly parabolic endomorphisms, i.e., endomorphisms $\varphi: E \rightarrow E$ such that, for each point $x \in D$,

$$
\varphi\left(E_{x, i}\right) \subset E_{x, i+1} .
$$

Analogously, we say that an endomorphism is non-strongly parabolic if it satisfies the weaker condition

$$
\varphi\left(E_{x, i}\right) \subset E_{x, i}
$$

and the sheaf of non-strongly parabolic endomorphisms is denoted ParEnd $(E)$. The tangent space at $[E]$ is isomorphic to $H^{1}(X$, ParEnd $(E))$. By the parabolic version of Serre duality,

$$
H^{1}(X, \operatorname{ParEnd}(E))^{*} \cong H^{0}(X, \operatorname{SParEnd}(E) \otimes K(D))
$$

and hence the Higgs field is an element of the cotangent space $T_{[E]}^{*} \mathcal{M}$, and the moduli space $\mathcal{M}(r, d, \alpha)$ of stable parabolic bundles is an open dense subset of the moduli 
$\mathcal{N}(r, d, \alpha)$ of parabolic Higgs bundles. The reader would like to see [Y, Y2, GGM] for references.

A trivial observation is that $\mathcal{M}(r, d, \alpha)$ is embedded in $\mathcal{N}(r, d, \alpha)$, just take null Higgs fields.

Define the determinant map from $\mathcal{N}(r, d, \alpha)$ to the cotangent $T^{*} \operatorname{Jac}^{d}(X)$, which is canonically isomorphic to $\operatorname{Jac}^{d}(X) \times H^{0}(X, K)$ :

$$
\begin{aligned}
\operatorname{det}: \mathcal{N}(r, d, \alpha) & \rightarrow \operatorname{Jac}^{d}(X) \times H^{0}(X, K), \\
(E, \Phi) & \mapsto\left(\Lambda^{r} E, \operatorname{tr} \Phi\right) .
\end{aligned}
$$

This is well defined because, since $\Phi$ is strongly parabolic, its trace actually lies in $H^{0}(X, K) \subset H^{0}(X, K(D))$. Let $\xi$ be a fixed line bundle of degree $d$. By definition, the fiber over $(\xi, 0)$ is called the moduli space of stable parabolic Higgs bundles with fixed determinant $\xi$, i.e.

$$
\mathcal{N}_{\xi}(r, d, \alpha):=\operatorname{det}^{-1}(\xi, 0) .
$$

Hence, for each $[(E, \Phi)] \in \mathcal{N}_{\xi}(r, d, \alpha)$, $\Phi$ is a traceless $K$-valued meromorphic endomorphism, with simple poles in $D$, and nilpotent residues respecting the parabolic filtration.

For fixed determinant $\xi$ it is

$$
\operatorname{dim} \mathcal{N}_{\xi}(r, d, \alpha)=2 \operatorname{dim} \mathcal{M}_{\xi}(r, d, \alpha)=2(g-1)\left(r^{2}-1\right)+n\left(r^{2}-r\right) .
$$

\section{Hitchin map AND SPECTRAL CURVES}

We continue now defining the Hitchin map and the Hitchin space for parabolic Higgs bundles. Let $S=\mathbb{V}(K(D))$ be the total space of the line bundle $K(D)$, let

$$
p: S=\underline{\operatorname{Spec}} \operatorname{Sym}^{\bullet}\left(K^{-1} \otimes \mathcal{O}_{X}(D)^{-1}\right) \longrightarrow X
$$

be the projection, and $x \in H^{0}\left(S, p^{*}(K(D))\right)$ be the tautological section. The characteristic polynomial of a Higgs field

$$
\operatorname{det}\left(x \cdot \mathrm{id}-p^{*} \Phi\right)=x^{r}+\tilde{s_{1}} x^{r-1}+\tilde{s_{2}} x^{r-2}+\cdots+\tilde{s_{r}}
$$

defines sections $s_{i} \in H^{0}\left(X, K^{i} D^{i}\right)$, such that $\tilde{s}_{i}=p^{*} s_{i}$ and $K^{i} D^{j}$ denotes the tensor product of the $i$ th power of $K$ with the $j$ th power of the line bundle associated to $D$.

We are assuming that $\Phi$ is strongly parabolic, therefore the residue at each point of $D$ is nilpotent. This implies that the eigenvalues of $\Phi$ vanish at $D$, i.e., for each $i>0$ the section $s_{i}$ belongs to the subspace $H^{0}\left(X, K^{i} D^{i-1}\right)$. We therefore define the Hitchin space as

$$
\mathcal{H}=H^{0}(K) \oplus H^{0}\left(K^{2} D\right) \oplus \cdots \oplus H^{0}\left(K^{r} D^{r-1}\right) .
$$

We also assume that $\Phi$ is traceless, i.e. $s_{1}=0$, and then define the traceless Hitchin space as

$$
\mathcal{H}_{0}=H^{0}\left(K^{2} D\right) \oplus \cdots \oplus H^{0}\left(K^{r} D^{r-1}\right) .
$$

Using Riemann-Roch, the parabolic Serre duality and the fact that $\operatorname{deg} K^{n} D^{n-1}<0$ we obtain the dimensions of $\mathcal{H}$ and $\mathcal{H}_{0}$. That is,

$$
\operatorname{dim} \mathcal{H}_{0}=(g-1)\left(r^{2}-1\right)+\frac{n\left(r^{2}-r\right)}{2},
$$


which is equal to the dimension of $\mathcal{M}_{\xi}$, and

$$
\operatorname{dim} \mathcal{H}=(g-1) r^{2}+1+\frac{n\left(r^{2}-r\right)}{2},
$$

which is equal to the dimension of $\mathcal{M}(r, d, \alpha)$.

Taking the characteristic polynomial of a Higgs field defines the Hitchin map

$$
h: \mathcal{N}(r, d, \alpha) \longrightarrow \mathcal{H}
$$

which we can restrict to the moduli space of fixed determinant

$$
h_{0}: \mathcal{N}_{\xi}(r, d, \alpha) \longrightarrow \mathcal{H}_{0}
$$

Given $s=\left(s_{1}, \ldots, s_{r}\right) \in \mathcal{H}$, with

$$
s_{i} \in H^{0}\left(K^{i} D^{i-1}\right) \subset H^{0}\left(K^{i} D^{i}\right),
$$

we think of $s_{i}$ as a section of $K^{i} D^{i}$, and then we define, as usual, the spectral curve $X_{s}$ in $S$ as the zero scheme of the section of $p^{*} K^{r} D^{r}$

$$
\psi=x^{r}+\widetilde{s}_{1} x^{r-1}+\widetilde{s}_{2} x^{r-2}+\cdots+\widetilde{s}_{r},
$$

where $\widetilde{s}_{i}=p^{*} s_{i}$. Denote by $\pi$ the restriction of the projection $p$ to the spectral curve $X_{s}$

$$
\pi: X_{s}=\underline{\operatorname{Spec}}\left(\operatorname{Sym}^{\bullet}\left(K^{-1} D^{-1} / \mathcal{I}\right)\right) \longrightarrow X
$$

where $\mathcal{I}$ is the ideal sheaf generated by the image of the homomorphism

$$
\begin{aligned}
K^{-r} D^{-r} & \longrightarrow \operatorname{Sym}^{\bullet}\left(K^{-1} D^{-1}\right) \\
\alpha & \longmapsto \alpha \sum_{i=0}^{r} s_{i}
\end{aligned}
$$

where we put $s_{0}=1$. From (3.3) it follows that

$$
\pi_{*} \mathcal{O}_{X_{s}}=\mathcal{O}_{X} \oplus K^{-1} D^{-1} \oplus K^{-2} D^{-2} \cdots \oplus K^{-r+1} D^{-r+1}
$$

Lemma 3.1. For $r \geq 2$ and $g \geq 2$ there is a dense open set in $\mathcal{H}$ whose spectral curve is smooth. The same holds for $\mathcal{H}_{0}$.

Proof. By remark 3.5 in BNR the set of sections $s$ such that the scheme $X_{s}$ is smooth, is open, and it is nonempty whenever $K^{r} D^{r-1}$ admits a section without multiple zeros. It is known (IV Corollary $3.2[\mathrm{H}]$ ) that, if $\operatorname{deg}\left(K^{r} D^{r-1}\right) \geq 2 g+1$, then $K^{r} D^{r-1}$ is very ample. It follows that, if $g \geq 2$, the line bundle $K^{r} D^{r-1}$ has sections without multiple zeros.

For $X_{s}$ smooth, the short exact sequences

$$
0 \longrightarrow T_{p} \cong p^{*} K(D) \longrightarrow T_{S} \longrightarrow p^{*} T_{X} \longrightarrow 0,
$$

where $T_{p}$ denotes the relative tangent bundle for the projection $p: S \longrightarrow X$, and

$$
\left.0 \longrightarrow T_{X_{s}} \longrightarrow T_{S}\right|_{X_{s}} \longrightarrow N_{X_{s} / S} \longrightarrow 0
$$

give

$$
N_{X_{s} / S} \cong K_{X_{s}} \otimes \pi^{*} D
$$

On the other hand,

$$
\left.N_{X_{s} / S} \cong \mathcal{O}\left(X_{s}\right)\right|_{X_{s}}=\left.p^{*}\left(K^{r} D^{r}\right)\right|_{X_{s}}=\pi^{*}\left(K^{r} D^{r}\right),
$$

and the ramification line bundle of the projection $\pi: X_{s} \rightarrow X$ is

$$
\mathcal{O}(R)=K_{X_{s}} \otimes \pi^{*} K^{-1}=\pi^{*} K^{r-1} D^{r-1} .
$$


The section

$$
\frac{\partial \psi}{\partial x}=r x^{r-1}+(r-1) \widetilde{s}_{1} x^{r-2}+\cdots+\widetilde{s}_{r-1} \in H^{0}\left(S, p^{*} K^{r-1} D^{r-1}\right),
$$

when restricted to $X_{s}$, gives a section of $\mathcal{O}(R)$, and its scheme of zeroes is exactly the ramification divisor $R$.

Lemma 3.2. If $X_{s}$ is smooth, then the fiber $h^{-1}(s)$ is isomorphic to

$$
\operatorname{Prym}\left(X_{s} / X\right)=\left\{L \in \operatorname{Pic}(X): \operatorname{det} \pi_{*} L \cong \xi\right\}
$$

Proof. Since $X_{s}$ is smooth, it is reduced, and hence the vector bundle $E$ is of the form $\pi_{*} L$ with $L$ an element of $\operatorname{Prym}\left(X_{s} / X\right)$. The morphism $\Phi$ is given by multiplication by the tautological section $x$, and it only remains to show that the parabolic structure can be recovered from $\Phi$.

For each parabolic point $p \in D$ there is a Zariski open subset of $X$ where the vector bundle is of the form $\left.E\right|_{U}=\mathcal{O}_{U}[x] / I$ (seen as an $\mathcal{O}_{U}$-module), where

$$
I=\left(x^{r}+s_{2} x^{r-2}+\cdots+s_{r}\right)
$$

Recall that $s_{i}$ vanishes at $p$ for all $i$. Hence, the fiber of $E$ over $p$ is

$$
\left.E\right|_{p}=\mathbb{C}[x] /\left(x^{r}\right) .
$$

On the other hand $\left.\Phi\right|_{x}$ is given by multiplication by $x$, and hence it defines a full flag on $\left.E\right|_{p}$, which, by definition of parabolic Higgs bundle, must coincide with the parabolic filtration. Therefore, the parabolic structure is recovered by $\Phi$.

Remark 3.3. Although this is written under the assumption of $\Phi$ being strongly parabolic it also works for non-strongly parabolic Higgs field. Obtaining, in such a case, the Jacobian of the spectral curve instead of the Prym variety (see [LM]).

\section{The NiLPotent CONE}

The fiber $h^{-1}(0)$ is called the nilpotent cone, and it is a Lagrangian subscheme [GGM, Thm. 3.14]. It follows that $h_{0}^{-1}(0)$ is also a Lagrangian subscheme. Indeed, it is obviously isotropic, since it is a subscheme of an isotropic variety, and the dimension of its components is $\operatorname{dim} h^{-1}(0)-g$, because it is the preimage in $h^{-1}(0)$ of $(\xi, 0) \in T^{*} \operatorname{Jac}^{d}(x)$ under the determinant map, and all the fibers of this map are isomorphic. We call it the nilpotent cone for traceless fields.

Note that, if $(E, \Phi)$ belongs to the nilpotent cone, then $\Phi$ is a nilpotent homomorphism (hence the name). The moduli space of parabolic bundles is embedded in the nilpotent cone as an irreducible component, via the map $E \mapsto(E, 0)$. In general there are other irreducible components corresponding to nonvanishing nilpotent homomorphisms, and we are going to see that the moduli space of parabolic bundles can be characterized as the unique irreducible component of the nilpotent cone which does not admit a non-trivial $\mathbb{C}^{*}$-action. To show that it does not admit a nontrivial $\mathbb{C}^{*}$-action, it is enough to show that $H^{0}\left(\mathcal{M}_{\xi}, T_{\mathcal{M}_{\xi}}\right)=0$, since a non-trivial $\mathbb{C}^{*}$-action produces a non-zero vector field.

In [NR] Narasimhan and Ramanan prove that if $\mathcal{S U}_{\xi}$ is the moduli space of stable vector bundles with fixed determinant, then $H^{0}\left(\mathcal{S U}_{\xi}, T_{\mathcal{S} \mathcal{U}_{\xi}}\right)=0$. We prove the following

Proposition 4.1. Assume that the parabolic weights are generic (so that parabolic semistable implies parabolic stable, and the moduli space is smooth) and small enough 
so that the stability of the parabolic Higgs bundle is equivalent to the stability of the underlying vector bundle. Then, $H^{0}\left(\mathcal{M}_{\xi}, T_{\mathcal{M}_{\xi}}\right)=0$.

Proof. Let $f: \mathcal{M}_{\xi} \rightarrow \mathcal{S U}_{\xi}$ be the morphism sending a parabolic bundle to the underlying vector bundle. Since we are assuming that the weights are small enough, the stability of a parabolic bundle coincides with the stability of the underlying vector bundle. Hence this morphism is well defined and it is a projection with fibers, fixing the weights, consisting of flag varieties giving us the filtration over each marked point.

The exact sequence over $\mathcal{M}_{\xi}$

$$
0 \rightarrow T_{f} \rightarrow T_{\mathcal{M}_{\xi}} \rightarrow f^{*}\left(T_{\mathcal{S U}_{\xi}}\right) \rightarrow 0
$$

gives an isomorphism $H^{0}\left(\mathcal{M}_{\xi}, T_{\mathcal{M}_{\xi}}\right) \cong H^{0}\left(\mathcal{M}_{\xi}, T_{f}\right)$, because $T_{\mathcal{S} \mathcal{U}_{\xi}}$ has no global sections [NR, Theorem 1] and $f$ is projective. The projection formula implies

$$
H^{0}\left(\mathcal{M}_{\xi}, T_{f}\right) \cong H^{0}\left(\mathcal{S \mathcal { U } _ { \xi }}, f_{*}\left(T_{f}\right)\right) \text {. }
$$

We claim that $f_{*}\left(T_{f}\right)=\operatorname{End}_{0}\left(\mathcal{E}_{x}\right)$, where $\mathcal{E}_{x}$ is the universal vector bundle on $\mathcal{S U}_{\xi} \times X$ restricted to the slice $\mathcal{S U}_{\xi} \times\{x\}$. Indeed, the fiber of $f$ over a point corresponding to $E$ is the flag variety of the vector space $E_{x}$, the fiber of $E$ over the point $x \in X$. This flag variety is $\mathrm{SL}\left(E_{x}\right) / B$ where $B$ is a Borel subgroup of $\mathrm{SL}\left(E_{x}\right)$. We have $H^{0}\left(\mathrm{SL}\left(E_{x}\right) / B, T_{\mathrm{SL}\left(E_{x}\right) / B}\right)=\mathfrak{s l}\left(E_{x}\right)$ ([A, Sec 4.8, Thm 2]), and therefore $f_{*}\left(T_{f}\right)=\operatorname{End}_{0}\left(\mathcal{E}_{x}\right)$.

Note that the universal vector bundle exists because $r$ and $\operatorname{deg}(\xi)$ are coprime. Therefore,

$$
H^{0}\left(\mathcal{S U}_{\xi}, f_{*}\left(T_{f}\right)\right) \cong H^{0}\left(\mathcal{S U}_{\xi}, \operatorname{End}_{0}\left(\mathcal{E}_{x}\right)\right)=0
$$

where the last equality is in [NR, Theorem 2].

We can adapt Simpson's Lemma 11.9 in [S] to the parabolic situation with non zero degree, and then we obtain the following

Lemma 4.2. Let $(E, \Phi)$ be a parabolic Higgs bundle in the nilpotent cone, with $\Phi \neq 0$. Consider the standard $\mathbb{C}^{*}$-action sending $(E, \Phi)$ to $(E, t \Phi)$. Assume that $(E, \Phi)$ is a fixed point. Then there is another Higgs bundle $\left(E^{\prime}, \Phi^{\prime}\right)$ in the nilpotent cone, not isomorphic to $(E, \Phi)$ such that $\lim _{t \rightarrow \infty}\left(E^{\prime}, t \Phi^{\prime}\right)=(E, \Phi)$.

These two results combine in the following

Proposition 4.3. Let $\mathcal{N}_{\xi}(r, d, \alpha)$ be the moduli space of parabolic Higgs bundles with fixed determinant $\xi$ over a compact Riemann surface $X$ of genus $g \geq 2$, and let $h_{0}$ be the corresponding Hitchin map. Then, there is only one component inside the nilpotent cone $h_{0}^{-1}(0)$ which admits no nontrivial $\mathbb{C}^{*}$-action, and it is the moduli space of parabolic bundles $\mathcal{M}_{\xi}(r, d, \alpha)$ with fixed determinant $\xi$ over the compact Riemann surface $X$.

Proof. Recall that the moduli space $\mathcal{M}_{\xi}$ of parabolic bundles with fixed determinant is embedded naturally in the moduli space $\mathcal{N}_{\xi}$ of parabolic Higgs bundles with fixed determinant just by sending again $E \mapsto(E, 0)$. From Proposition 4.1 we know that $\mathcal{M}_{\xi}$ has no nontrivial $\mathbb{C}^{*}$-action and from dimensional computation it is one connected component of the nilpotent cone. It remains to check that there is no other connected component where there is no $\mathbb{C}^{*}$-action.

In the rest of the components, with nonzero Higgs field, we have a $\mathbb{C}^{*}$-action given by $(E, \Phi) \mapsto(E, t \Phi)$. And this action is nontrivial thanks to lemma 4.2, 


\section{The ORIGin of the Hitchin SPACE.}

We are going to identify the nilpotent cone among the fibers of the Hitchin map. For each point $s \in \mathcal{H}$ of the Hitchin space we have a spectral curve $X_{s} \subset S$ given by the spectral construction, and this gives us a family of curves on $S$ parameterized by $\mathcal{H}$. Let $s \in \mathcal{H}$ be a point such that the corresponding spectral curve $X_{s}$ is smooth. If we move the point $s$, the spectral curve $X_{s}$ is deformed. The Kodaira-Spencer map

$$
u: T_{s} \mathcal{H} \cong \mathcal{H} \rightarrow H^{1}\left(X_{s}, T_{X_{s}}\right)
$$

gives, for each vector in the tangent space $T_{s} \mathcal{H}$, the corresponding infinitesimal deformation of the curve, which is described by an element of $H^{1}\left(X_{s}, T_{X_{s}}\right)$. We will also consider the restriction of $u$ to the traceless Hitchin space obtaining another Kodaira-Spencer map

$$
u_{0}: T_{s} \mathcal{H}_{0} \longrightarrow H^{1}\left(X_{s}, T_{X_{s}}\right) .
$$

A tangent vector at a point on the restricted Hitchin space $s \in \mathcal{H}_{0}$ defined by the standard action of $\mathbb{C}^{*}$ is contained in the kernel of the restricted Kodaira-Spencer map, because the standard $\mathbb{C}^{*}$-action does not change the isomorphism class of the spectral curve. On the other hand, in Proposition 5.2 we show that the dimension of the kernel of $u_{0}$ is one. Therefore, the direction defined by the standard $\mathbb{C}^{*}$-action coincides with the kernel of $u_{0}$.

By lemma 3.2, the fiber of the Hitchin map $h_{0}$ over a point $s$ corresponding to a smooth spectral curve $X_{s}$ is isomorphic to the Prim variety $P_{s}=\operatorname{Prym}\left(X_{s} / X\right)$. Therefore, if we move $s$ we will get a deformation of $P_{s}$. In particular, we get a Kodaira-Spencer map between the infinitesimal deformations

$$
v_{0}: T_{s} \mathcal{H}_{0} \longrightarrow H^{1}\left(P_{s}, T_{P_{s}}\right)
$$

The tangent vector defined by any $\mathbb{C}^{*}$-action on $\mathcal{H}_{0}$ which lifts to $\mathcal{N}_{\xi}(r, d, \alpha)$ is in the kernel of $v_{0}$. Indeed, if the action lifts, it provides an isomorphism among the fibers over the orbit of the action, hence the infinitesimal deformation of $P_{s}$ has to be zero. We will see that a non-trivial the curve $X_{s}$ produces a non-trivial deformation of $P_{s}$, and then we obtain that the tangent vector defined by any $\mathbb{C}^{*}$-action which lifts to $\mathcal{N}_{\xi}(r, d, \alpha)$ is in the kernel of $u_{0}$.

We use the following

Lemma 5.1. If $X_{s}$ is smooth, then there are natural isomorphisms

$$
T_{s} \widetilde{\mathcal{H}} \cong H^{0}\left(X_{s}, \pi^{*}\left(K^{r} D^{r}\right)\right) \cong H^{0}\left(X_{s}, N_{X_{s} / S}\right) .
$$

Proof. Using the isomorphisms (3.6) and (3.4) and the projection formula, we have

$$
\begin{aligned}
H^{0}\left(X_{s}, N_{X_{s} / S}\right) & =H^{0}\left(X_{s}, \pi^{*}\left(K^{r} D^{r}\right)\right)=H^{0}\left(X, K^{r} D^{r} \otimes \pi_{*} \mathcal{O}_{X_{s}}\right)= \\
& =H^{0}\left(X, \oplus_{i=1}^{r}\left(K^{i} D^{i}\right)\right)=\widetilde{\mathcal{H}} \cong T_{s} \widetilde{\mathcal{H}}
\end{aligned}
$$

The objective now is to calculate the kernels of $u$ and $u_{0}$. There are some elements in $H^{0}\left(X_{s}, N_{X_{s} / S}\right)$ that are clearly in the kernel. For instance, let $\lambda \in H^{0}\left(X, \mathcal{O}_{X}\right) \cong$ $\mathbb{C}$, and denote a point in $X_{s} \subset S$ by $(\omega, v)$, where $\omega$ is a point in $X$ and $x$ is a point in the fiber of $S$ over $\omega$. Then the deformation sending $(\omega, v)$ to $\left(\omega, e^{\lambda} v\right)$ clearly does not change the isomorphism class of $X_{s}$. In fact, this is the deformation produced by the standard $\mathbb{C}^{*}$-action, and it is clearly in the kernel of the Kodaira-Spencer map $u_{0}$. 
Furthermore, for any $\alpha \in H^{0}(X, K(D))$, sending $(\omega, v)$ to $(\omega, v+\alpha(\omega))$ also preserves the isomorphism class of $X_{s}$. The deformations defined in this way do not preserve the condition $0=\operatorname{tr}(\Phi)\left(=s_{1}\right)$, and hence they are in the kernel of $u$, but not in $T_{s} \mathcal{H}_{0}$. The following proposition says that these two constructions describe the kernels.

Proposition 5.2. The kernel of the Kodaira-Spencer map $\widetilde{u}$ is given by the following exact sequence

$$
0 \longrightarrow H^{0}\left(X, K(D) \oplus \mathcal{O}_{X}\right) \longrightarrow T_{s} \widetilde{\mathcal{H}} \stackrel{\widetilde{u}}{\longrightarrow} H^{1}\left(X_{s}, T_{X_{s}}\right)
$$

so it has dimension $g+n+1$ (recall $n=\operatorname{deg} D$ ). If we fix the determinant, the restriction of the Kodaira-Spencer map provides an exact sequence

$$
0 \longrightarrow H^{0}\left(X, \mathcal{O}_{X}\right) \longrightarrow T_{s} \widetilde{\mathcal{H}}_{0} \stackrel{\widetilde{u}_{0}}{\longrightarrow} H^{1}\left(X_{s}, T_{X_{s}}\right),
$$

and hence $\operatorname{dim} \operatorname{Ker} \widetilde{u}_{0}=1$. If we restrict the Kodaira-Spencer map to $\mathcal{H}_{0}$ then we have

$$
0 \longrightarrow H^{0}\left(X, \mathcal{O}_{X}\right) \longrightarrow T_{s} \mathcal{H}_{0} \stackrel{u_{0}}{\longrightarrow} H^{1}\left(X_{s}, T_{X_{s}}\right),
$$

and hence $\operatorname{dim} \operatorname{Ker} \widetilde{u}_{0}=1$.

Proof. Consider the following diagram, constructed using (3.6)

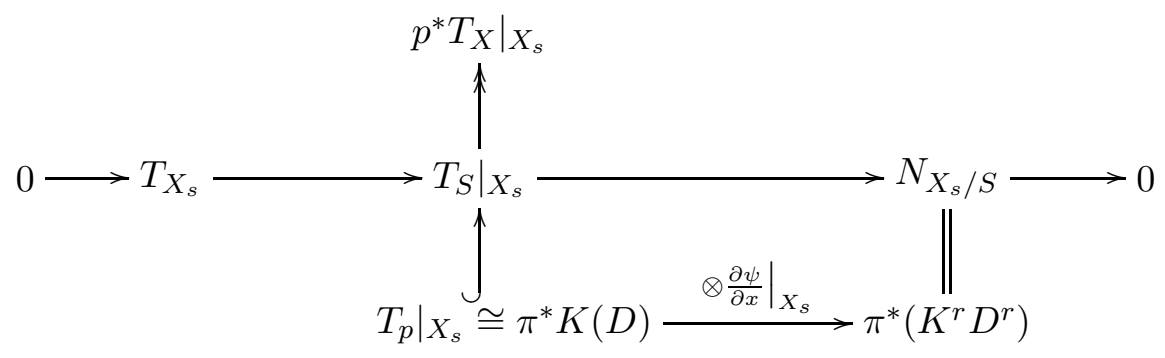

where $T_{p}$ denotes the relative tangent bundle for the projection $p: S \longrightarrow X$, as in section 3. Note that the diagram is well defined, since $\left.\frac{\partial \psi}{\partial x}\right|_{X_{s}}$ is a section of

$$
\pi^{*}\left(K^{r-1} D^{r-1}\right) \cong \mathcal{O}(R)
$$

(cf.(3.7)). The diagram is commutative because the zero scheme of the two morphisms between the line bundles $\left.T_{p}\right|_{X_{s}}$ and $N_{X_{s} / S}$ are the same, namely the ramification divisor, hence the maps differ by a scalar, but this scalar can be absorbed in the projection $\left.T_{S}\right|_{X_{s}} \rightarrow N_{X_{s} / S}$.

Since the tangent line bundle $T_{X}$ has negative degree, $H^{0}\left(X_{s},\left.p^{*} T_{X}\right|_{X_{s}}\right)=0$. Therefore, the middle column in diagram (5.1) gives

$$
H^{0}\left(X_{s},\left.T_{S}\right|_{X_{s}}\right) \cong H^{0}\left(X_{s},\left.T_{p}\right|_{X_{s}}\right) .
$$

On the other hand,

$$
\begin{gathered}
H^{0}\left(X_{s},\left.T_{p}\right|_{X_{s}}\right) \cong H^{0}\left(X_{s}, \pi^{*} K(D)\right) \cong \\
\cong H^{0}\left(X, K(D) \otimes \pi_{*} \mathcal{O}_{X_{s}}\right) \cong H^{0}\left(X, K(D) \oplus \mathcal{O}_{X}\right)
\end{gathered}
$$

which, together with lemma 5.1, transforms the long exact sequence given by the middle row of diagram (5.1),

$$
0 \rightarrow H^{0}\left(X_{s}, T_{X_{s}}\right) \rightarrow H^{0}\left(X_{s},\left.T_{S}\right|_{X_{s}}\right) \rightarrow H^{0}\left(X_{s}, N_{X_{s} / S}\right) \rightarrow H^{1}\left(X_{s}, T_{X_{s}}\right),
$$

into

$$
0 \longrightarrow H^{0}\left(X, K(D) \oplus \mathcal{O}_{X}\right) \longrightarrow T_{s} \widetilde{\mathcal{H}} \stackrel{u}{\longrightarrow} H^{1}\left(X_{s}, T_{X_{s}}\right),
$$


where we have used $T_{s} \widetilde{H} \cong H^{0}\left(X_{s}, \pi^{*}\left(K^{r} D^{r}\right)\right)$.

Now we restrict the Kodaira-Spencer map $u$ to $T_{s} \widetilde{\mathcal{H}}_{0}$. Using the isomorphism $H^{0}\left(X_{s}, \pi^{*}\left(K^{r} D^{r}\right)\right) \cong H^{0}\left(X, \oplus_{i=1}^{r}\left(K^{i} D^{i}\right)\right)$ (cf. proof of Lemma 5.1), an element of this group is written as

$$
\widetilde{a}_{0} x^{r}+\widetilde{a}_{1} x^{r-1}+\cdots+\widetilde{a}_{r}
$$

with $a_{i} \in H^{0}\left(X, K^{i} D^{i}\right), a_{0}=1$ and $\widetilde{a}_{i}=\pi^{*} a_{i}$.

On the other hand, an element of $H^{0}\left(X, K(D) \oplus \mathcal{O}_{X}\right)$ can be written as

$$
\widetilde{b}_{1}+\widetilde{b}_{0} x
$$

with $b_{i} \in H^{0}\left(X, K^{i} D^{i}\right)$ and $\widetilde{b}_{i}=\pi^{*} b_{i}$.

The map $\theta$ from one to another is $H^{0}\left(\left.\otimes \frac{\partial \psi}{\partial x}\right|_{X_{s}}\right)$. A short calculation (using $\left.\psi\right|_{X_{s}}=$ $0)$ gives

$$
\widetilde{\theta}\left(\widetilde{b}_{1}+\widetilde{b}_{0} x\right)=\sum_{i=1}^{r}\left((r-i+1) \widetilde{s}_{i-1} \widetilde{b}_{1}-i \widetilde{s}_{i} \widetilde{b}_{0}\right) x^{r-i}
$$

The subspace $\widetilde{\mathcal{H}}_{0} \subset \widetilde{\mathcal{H}}$ is the zero locus of the trace map sending $\left(s_{1}, \ldots, s_{r}\right)$ to $s_{1}$. Then we have a commutative diagram



where $p_{1}$ is projection to the first summand.

Now, if $s \in \widetilde{\mathcal{H}}_{0}$, then $s_{1}=0$, and using the explicit formula (5.2), we obtain

$$
(d(\operatorname{tr}) \circ \widetilde{\theta})\left(\widetilde{b}_{1}+\widetilde{b}_{0} x\right)=r \widetilde{b}_{1},
$$

and hence the following diagram is commutative

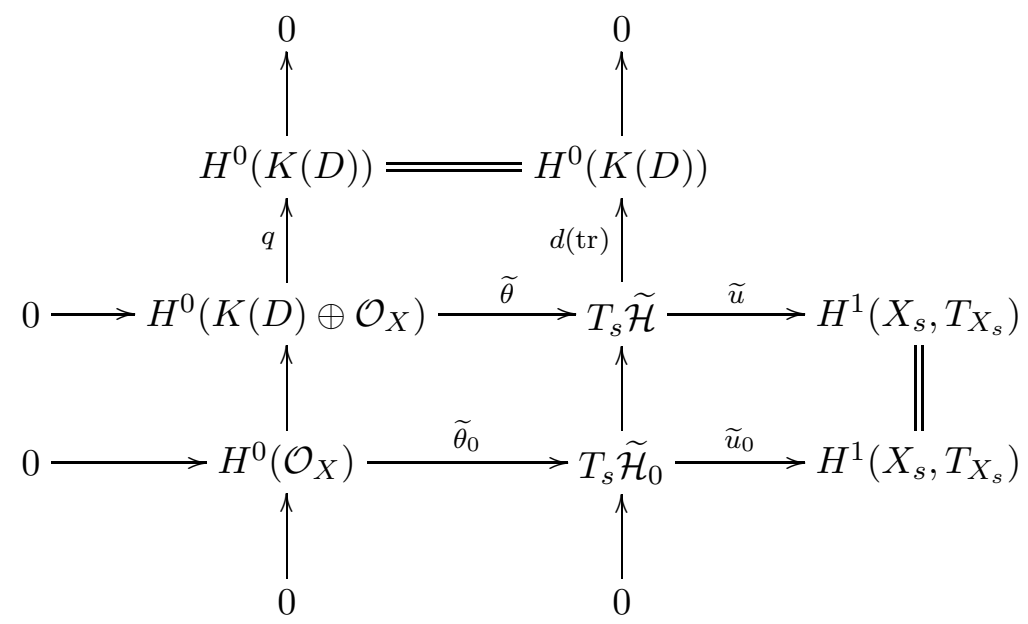

where $q$ is projection to the first summand followed by multiplication by $r$. The top row is the second exact sequence in the statement of the proposition.

Finally, if the spectral curve corresponds to a strongly parabolic homomorphism, i.e. $s=\left(s_{1}, \ldots, s_{r}\right) \in \mathcal{H}_{0}$ with

$$
s_{i} \in H^{0}\left(K^{i} D^{i-1}\right) \subset H^{0}\left(K^{i} D^{i}\right),
$$


then the image of $\widetilde{\theta}_{0}$ lies in

$$
H^{0}\left(X, \oplus_{i=2}^{r}\left(K^{i} D^{i-1}\right)\right) \cong T_{s} \mathcal{H}_{0}
$$

and therefore we get a commutative diagram

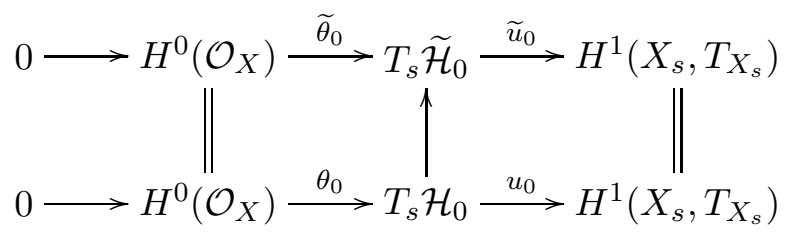

Proposition 5.3. Let $g: \mathbb{C}^{*} \times \mathcal{H}_{0} \longrightarrow \mathcal{H}_{0}$ be an action, having exactly one fixed point, and admitting a lift to $\mathcal{N}_{\xi}(r, d, \alpha)$. Then this fixed point is the origin of $\mathcal{H}_{0}$.

Proof. The proof is the same as in [BG, Proposition 5.1], so we only give a sketch, since the details can be found there.

Let $s \in \mathcal{H}_{0}$ be a point corresponding to a smooth spectral curve $X_{s}$ (it exists by Lemma 3.1). The tangent vector defined at this point by the standard action is contained in the kernel of the Kodaira-Spencer map $u_{0}$, since the standard action does not change the isomorphism class of the spectral curve. We are going to prove that the tangent vector defined by any action that lifts to $\mathcal{N}_{\xi}(r, d, \alpha)$ is also in the kernel of the Kodaira-Spencer map.

Let $J=\operatorname{Jac}(X), J_{s}=\operatorname{Jac}\left(X_{s}\right)$, and $P_{s}=\operatorname{Prym}\left(X_{s} / X\right)$. There is an étale covering

$$
\alpha: P_{s} \times J \rightarrow J_{s}
$$

sending $\left(L_{1}, L_{2}\right)$ to $L_{1} \otimes \pi^{*} L_{2}$.

Let $g: \mathbb{C}^{*} \times \mathcal{H}_{0} \rightarrow \mathcal{H}_{0}$ be an action. Its derivative gives a tangent vector $w$ at $s$, and the image of $w$ under the Kodaira-Spencer map produces an infinitesimal deformation of the spectral curve $u_{0}(w)=\eta_{1} \in H^{1}\left(X_{s}, T_{X_{s}}\right)$, its Jacobian $\eta_{2} \in$ $H^{1}\left(J_{s}, T_{J_{s}}\right)$, and the Prym variety $\eta_{3} \in H^{0}\left(P_{s}, T_{P_{s}}\right)$. We have homomorphisms

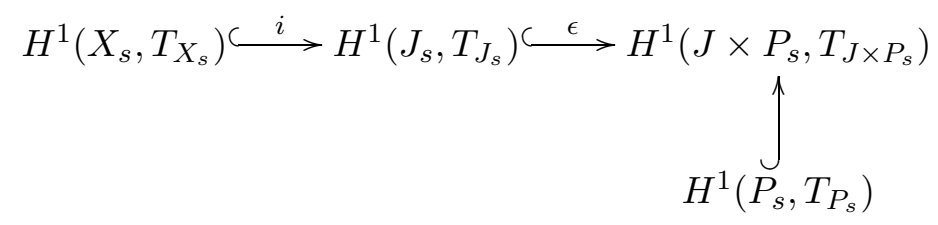

Indeed, a deformation of $X_{s}$ produces a deformation of $J_{s}$, and the corresponding homomorphism $i$ is injective because of the infinitesimal version of the classical Torelli theorem for curves (a non-zero deformation of a curve produces a non-zero deformation of its Jacobian). On the other hand, an infinitesimal deformation of $J_{s}$ produces an infinitesimal deformation of its étale covering $J \times P_{s}$. The image of the composition $\epsilon \circ i$ lies in $H^{1}\left(P_{s}, T_{P_{s}}\right)$, because a deformation of $J \times P_{s}$ induced by a deformation of $X_{s}$ (keeping $X$ constant) is induced by a deformation of the factor $P_{s}$.

We have $i\left(\eta_{1}\right)=\eta_{2}$ and $\epsilon\left(\eta_{2}\right)=\eta_{3}$. Recall that the fiber of $h_{0}$ over $s$ is canonically isomorphic to $P_{s}$. Now, if the action $g$ lifts to $\mathcal{N}_{\xi}(r, d, \alpha)$, all the fibers above the points of an orbit in $\mathcal{H}_{0}$ should be isomorphic, and therefore we should have $\eta_{3}=0$. By the injectivity of $\epsilon$ and $i$, this implies that $\eta_{1}=0$, i.e., $u_{0}(w)=0$, and then the tangent vector $w$ defined by the action $g$ is in the kernel of the Kodaira-Spencer map. 
Now let $g$ be an action of $\mathbb{C}^{*}$ on $\mathcal{H}_{0}$, which has exactly one fixed point, and which admits a lift to $\mathcal{N}_{\xi}(r, d, \alpha)$. There is a dense open set $U$ of $\mathcal{H}_{0}$ corresponding to smooth spectral curves (Lemma 3.1). In this open set, each orbit of $g$ is included in an orbit of the standard action, since for each point in this open set, the span of the tangent vector defined by the standard action coincides with the kernel of the Kodaira-Spencer map, and the tangent vector defined by $g$ is in this kernel. In particular, the origin, being the fixed point of the standard action, is a limiting point of all the orbits of $g$ in $U$, but it is not in the orbits of $g$ (because the fiber over the origin is not isomorphic to the fibers over $U$ ). The limiting points of a $\mathbb{C}^{*}$-action are fixed points, therefore the origin is a fixed point of $g$, and, by hypothesis is the only fixed point.

\section{Proof of MAIN THEOREM}

In this section we prove Theorem [1.1. Since we are going to apply the Torelli theorem for parabolic bundles in [BBB], we have to assume that the rank is 2 .

Proof of Theorem 1.1. The Hitchin map gives a surjective morphism from $\mathcal{N}_{\xi}(2, d, \alpha)$ to the vector space $\mathcal{H}$ called the Hitchin space. The fiber over the origin is called the nilpotent cone. Observe that $\mathcal{M}_{\xi}(2, d, \alpha) \subset h_{0}^{-1}(0)$ and has the same dimension as the fiber so, it is one connected component of this fiber. Actually, we will show that it is the unique irreducible component inside the nilpotent cone that does not admit a non-trivial $\mathbb{C}^{*}$-action. Therefore, using the Torelli theorem in $\mathrm{BBB}$, we recover the pointed curve. This is the idea used in [BG] to determine intrinsically $\mathcal{M}_{\xi}(2, d, \alpha)$ inside $\mathcal{N}_{\xi}(2, d, \alpha)$. We show that it also works in the parabolic situation.

Note that we are given only the isomorphism class of $\mathcal{N}_{\xi}(2, d, \alpha)$ so we do not have explicitly the Hitchin map.

Consider $Y$ an algebraic variety isomorphic to our moduli space $\mathcal{N}_{\xi}(2, d, \alpha)$ and the natural morphism $Y \rightarrow \operatorname{Spec}(\Gamma(Y))$. Since $Y$ is isomorphic to $\mathcal{N}_{\xi}(2, d, \alpha)$ and the fibers of the Hitchin map are compact by Lemma 3.2 it happens that the ring of global functions of $\mathcal{N}_{\xi}(2, d, \alpha)$ factorizes through the Hitchin map and $\operatorname{Spec}(\Gamma(Y)) \cong$ $\operatorname{Spec}\left(\Gamma\left(\mathcal{H}_{0}\right)\right)$ and hence

$$
\operatorname{Spec}(\Gamma(Y)) \cong \operatorname{Spec}\left(\Gamma\left(\mathcal{H}_{0}\right)\right) \cong \mathbb{C}\left[y_{1}, y_{2}, \ldots, y_{3(g-1)+n}\right] .
$$

The following diagram commutes,

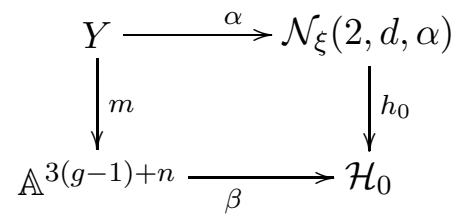

Let $g: \mathbb{C}^{*} \times \mathbb{A}^{3(g-1)+n} \longrightarrow \mathbb{A}^{3(g-1)+n}$ be a $\mathbb{C}^{*}$-action with exactly one fixed point $y$ and such that it admits a lift to $Y$. We know that it exists: take, for instance, the standard $\mathbb{C}^{*}$-action on $\mathcal{H}_{0}$ and apply it to $\mathbb{A}^{3(g-1)+n}$ through the isomorphism $\beta$.

We also know that such an action has as unique fixed point the origin by Proposition 5.3. Hence, the fiber over $y$ is isomorphic to the nilpotent cone $h_{0}^{-1}(0)$. It only remains to use Proposition 4.3 to identify $\mathcal{M}_{\xi}(2, d, \alpha)$ as the component inside the nilpotent cone which does not admit a nontrivial $\mathbb{C}^{*}$-action. By Theorem 3.2 in [BBB], from the isomorphism class of $\mathcal{M}_{\xi}(2, d, \alpha)$ we recover $(X, x)$ up to isomorphism. 


\section{REFERENCES}

[A] D. Akhiezer, Lie group actions in complex analysis. Aspects of Mathematics, E27. Friedr. Vieweg and Sohn, Braunschweig, 1995. viii+201 pp.

[BBB] V. Balaji, S. del Baño and I. Biswas, A Torelli type theorem for the moduli space of parabolic vector bundles over curves, Math. Proc. Cambridge Philos. Soc. 130 (2001), no. 2, 269-280.

[BG] I. Biswas and T. Gómez, A Torelli theorem for the moduli space of Higgs bundles on a curve, Quarterly Journal of Mathematics 54 (2003), 159-169.

[BNR] A. Beauville, M.S. Narasimhan and S. Ramanan, Spectral curves and the generalized theta divisor, J. reine angew. Math. 398 (1989), 169-179.

[GGM] O. García-Prada, P. B. Gothen and V. Muñoz, Betti numbers of the moduli space of rank 3 parabolic Higgs bundles, Memoirs of the American Mathematical Society, vol. 187, 879 (2007).

[MS] V.B. Mehta and C.S. Seshadri, Moduli of vector bundles on curves with parabolic structures, Math. Ann. 248, (1980) 205-239.

[H] R. Hartshorne, Algebraic Geometry Graduate Texts in Mathematics, No. 52. SpringerVerlag, New York-Heidelberg, 1977.

[H1] N.J. Hitchin, The self-duality equations on a Riemann surface, Proc. London Math. Soc. (3) 55 (1987), no. 1, 59-126.

[H2] N.J. Hitchin, Stable bundles and integrable systems, Duke Math. J. 54 (1987), no. 1, 91-114.

[LM] M. Logares and J. Martens, Moduli of parabolic Higgs bundles and Atiyah algebroids, to appear.

[MN] D. Mumford and P. Newstead, Periods of a moduli space of bundles on curves. Amer. J. Math. 901968 1200-1208.

[NR] M.S. Narasimhan and S. Ramanan, Deformations of the moduli space of vector bundles over an algebraic curve, Ann. Math. (2) 101 (1975), 391-417.

[NR2] M.S. Narasimhan and S. Ramanan, Generalized Prym varieties as fixed points, Jour. Indian Math. Soc. 39 (1975), 1-19.

[S] C. Simpson, Moduli of representations of the fundamental group of a smooth projective variety II, Publi. Math I.H.E.S 80 (1995), 5-79.

[W] A. Weil, Generalisation des fonctions abeliennes, J. Math. Pures Appl. 17 (1938) 47-87.

[Y] K.Yokogawa, Infinitesimal deformation of parabolic Higgs sheaves, Int. J. Math. 6 (1995) 125-148.

[Y2] K.Yokogawa, Compactification of moduli of parabolic sheaves and moduli of parabolic Higgs sheaves, J. Math. Kyoto Univ. 33 (1993) 451-504.

Instituto de Ciencias Matemáticas (CSIC-UAM-UC3M-UCM), Serrano 113bis, 28006 Madrid, Spain; and Facultad de Ciencias Matemáticas, Universidad Complutense de MADRID, 28040 MADRID, SPAIN

E-mail address: tomas.gomez@mat.csic.es

Departamento de Matematica Pura, Facultade de Ciencias, Rua do Campo Alegre 687, 4169-007 Porto Portugal

E-mail address: mlogares@fc.up.pt 